\title{
Intracellular trafficking*
}

\author{
Barth D. Grant ${ }^{\S}$, Miyuki Sato, Department of Molecular Biology and \\ Biochemistry, Rutgers University, Piscataway, NJ 08854 USA
}

\section{Table of Contents}

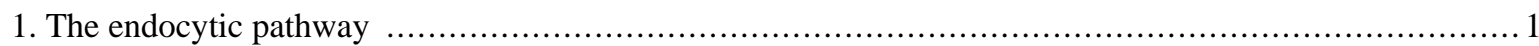

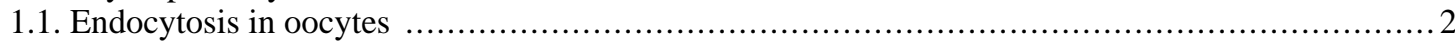

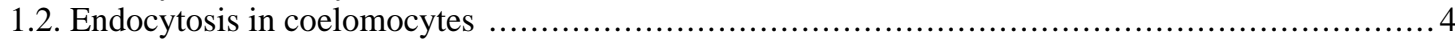

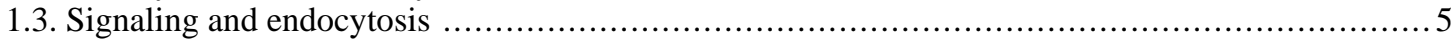

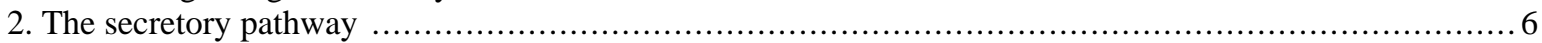

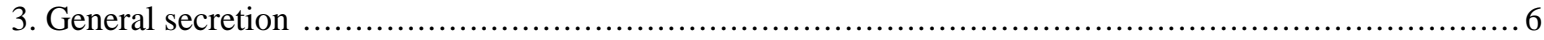

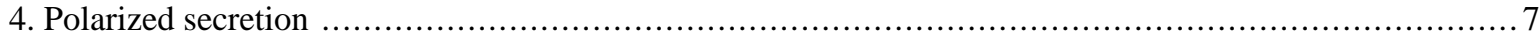

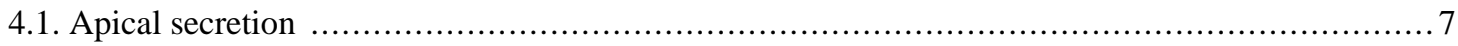

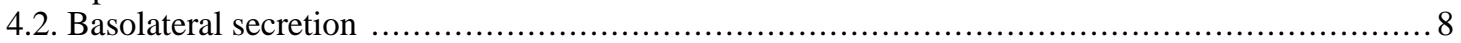

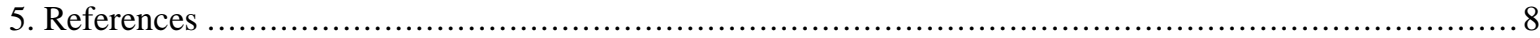

\begin{abstract}
Studies in $C$. elegans have begun to reveal new components and new mechanisms associated with intracellular membrane traffic in a variety of cell types. The worm benefits from many of the advantages of yeast as a genetically tractable organism for these kinds of studies while offering the unique opportunity to probe how these pathways have been extended and modified in the context of a multicellular animal undergoing development to produce diverse cell types such as muscles, nerves, and polarized epithelia. This review summarizes recent work elucidating endocytic pathways, primarily in the worm germ line and coelomocytes, and also touches on diverse studies of secretion, especially in ectodermal cells of epithelial character.
\end{abstract}

\section{The endocytic pathway}

Endocytosis is the vesicle-mediated process used by all cells to internalize extracellular macromolecules, plasma membrane lipids, and plasma membrane proteins (Figure 1). There are several endocytic pathways that utilize different mechanisms to internalize portions of the plasma membrane. The best studied endocytosis pathway in worms (Fares and Grant, 2002) and mammalian cells (Brodsky et al., 2001) is the clathrin-coated pit pathway (Figure 2). Many receptors and their associated ligands cluster into clathrin-coated pits by association with clathrin

*Edited by James M. Kramer and Donald G. Moerman. Last revised March 10, 2005. Published January 21, 2006. This chapter should be cited as: Grant, B. D. and Sato, M. Intracellular trafficking (January 21, 2006), WormBook, ed. The C. elegans Research Community, WormBook, doi/10.1895/wormbook.1.77.1, http://www.wormbook.org.

Copyright: () 2006 Barth D. Grant and Miyuki Sato. This is an open-access article distributed under the terms of the Creative Commons Attribution License, which permits unrestricted use, distribution, and reproduction in any medium, provided the original author and source are credited.

${ }^{\S}$ To whom correspondence should be addressed. E-mail: grant@biology.rutgers.edu 
adaptor proteins such as the four-subunit complex AP2. Clathrin adaptors in turn bind to the clathrin lattice which is thought to provide the force required to deform the membrane into a curved bud. The large GTPase dynamin is then involved in pinching off the coated pit to form a clathrin-coated vesicle. Such vesicles are then uncoated by the chaperone hsc70 and the DNA-J domain co-chaperone auxillin. Uncoated endocytic vesicles then fuse with one another and with early endosomes in a reaction requiring the small GTPase Rab5. In early endosomes some ligand-receptor complexes dissociate due to the reduced $\mathrm{pH}$ of the endosomal lumen. Many receptors then recycle to the plasma membrane either directly or indirectly via recycling endosomes. Many ligands do not recycle but instead are transported from early to late endosomes and eventually to lysosomes for degradation. Early to late endosome transport may be mediated by small vesicular intermediates, or may be a maturation process whereby early endosomes lose components through recycling pathways and gain components through fusion with vesicles derived from the secretory pathway. Late endosomes are then thought to fuse with pre-lysosomes to form "hybrid" organelles, which mature back into lysosomes through sorting and fission.

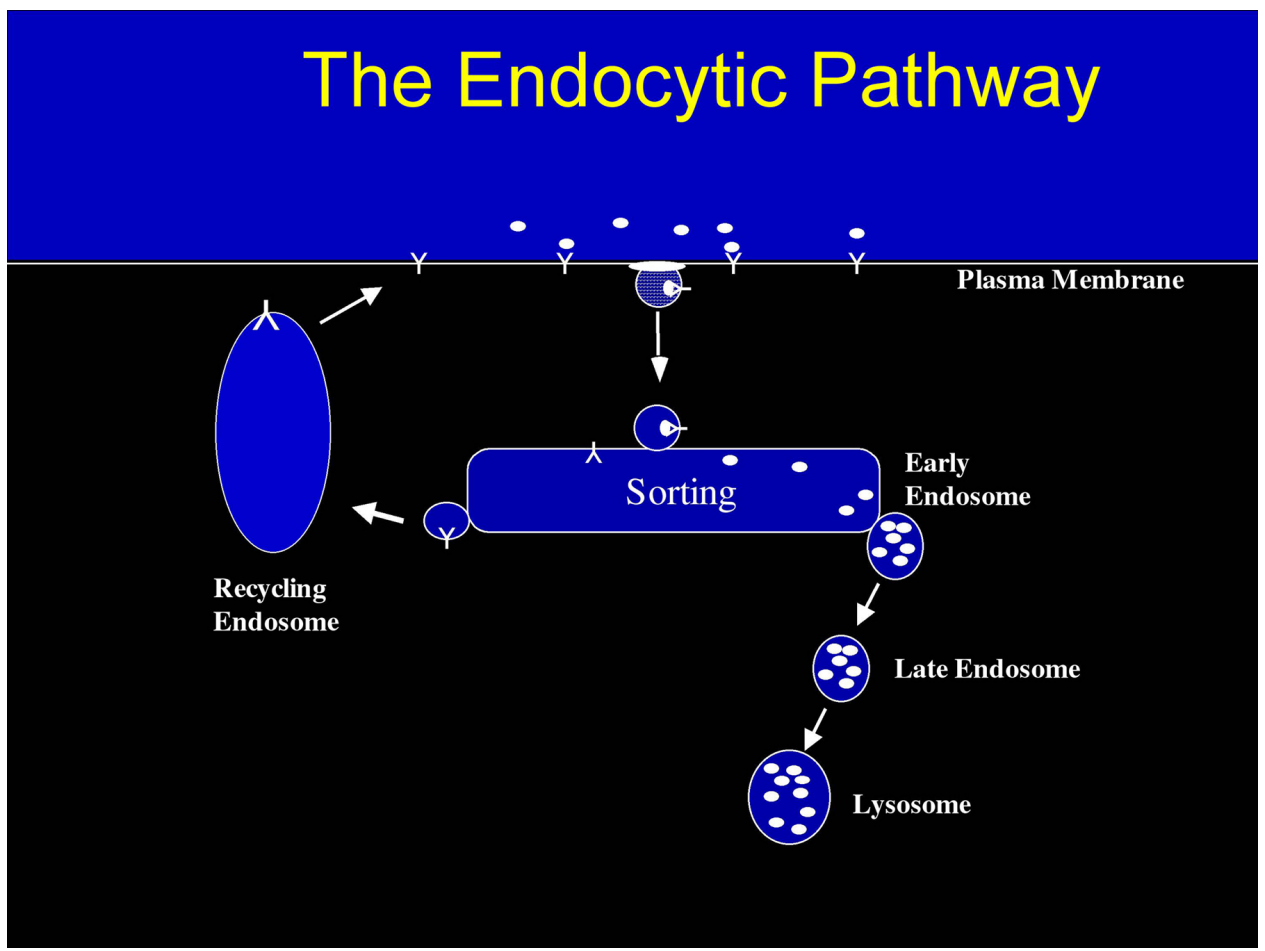

Figure 1. General model of the endocytic pathway. Cargo molecules are endocytosed and targeted to early endosomes. Some cargos are further transported to lysosomes through late endosomes. Others are recycled back to the plasma membrane via the recycling pathway.

\subsection{Endocytosis in oocytes}

One important model for mechanistic studies of endocytosis in C. elegans focuses on oocytes, which internalize huge quantities of yolk proteins and their associated lipids by clathrin-mediated endocytosis (Grant and Hirsh, 1999). This process is most easily followed by fluorescence microscopy using strains that express transgenes encoding the major yolk protein YP-170 (aka VIT-2) fused to GFP (Figure 3; Grant and Hirsh, 1999). YP170-GFP is synthesized in the intestine of adult hermaphrodites and is secreted basolaterally into the body cavity (pseudocoelom). YP170-GFP, like endogenous yolk, is a cholesterol binding/transport protein related to human ApoB-100, the major protein component of serum low-density lipoprotein (LDL). The yolk receptor in $C$. elegans is RME-2, an LDL-receptor related molecule expressed specifically in the oocytes (Figure 3; Grant and Hirsh, 1999). RME-2 contains a typical NPXY internalization motif in its intracellular domain that is known to direct other members of the LDL-receptor family into clathrin-coated pits. Trafficking of yolk and yolk receptors also depends critically upon the activities of the endocytic Rab proteins RAB-5, RAB-7, and RAB-11, known modulators of endocytosis in all eukaryotes (Grant and Hirsh, 1999). Thus yolk uptake provides a genetically tractable system for the study of clathrin and Rab dependent transport processes. 


\section{Clathrin-dependent endocytosis}

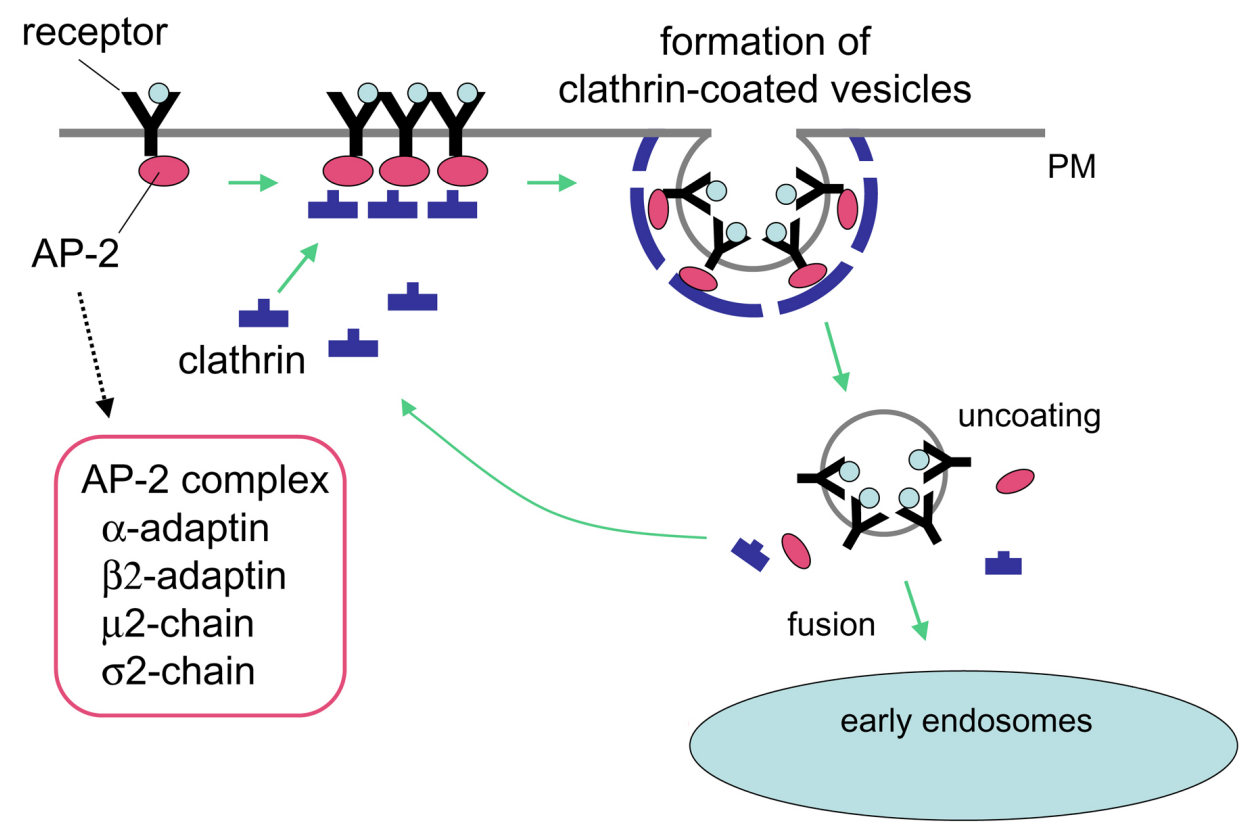

Figure 2. Mechanism of clathrin-dependent endocytosis. Clathrin and cargo molecules are assembled into clathrin-coated pits on the plasma membrane together with an adaptor complex called AP-2 that links clathrin with transmembrane receptors, concluding in the formation of mature clathrin-coated vesicles (CCVs). CCVs are then actively uncoated and transported to early/sorting endosomes.

\section{YP170::GFP endocytosis by oocytes}

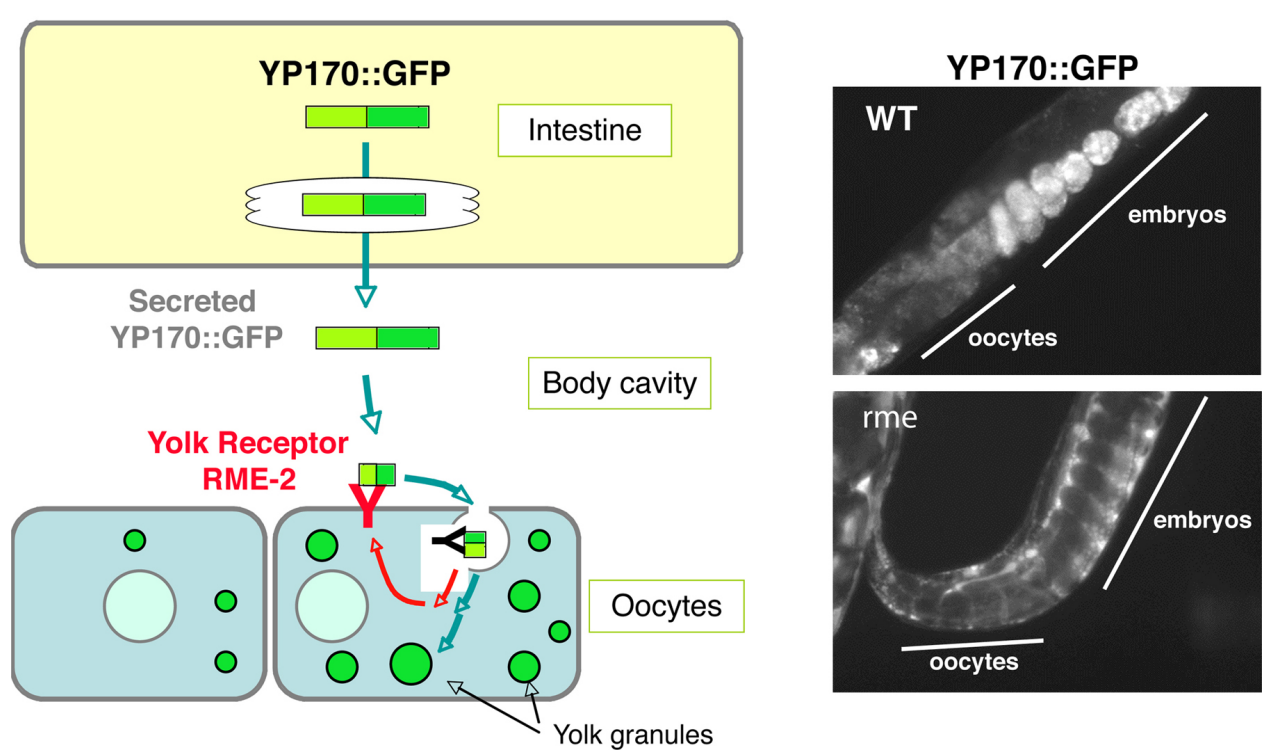

Figure 3. YP170::GFP endocytosis by oocytes. YP170::GFP is synthesized in the intestine and secreted into the body cavity from which it is endocytosed by oocytes. RME-2 is the yolk receptor expressed in oocytes. Fluorescent micrographs of wild-type and typical rme mutant worms expressing YP170::GFP are shown.

Screens for mutants defective in YP170-GFP uptake by oocytes identified 11 rme genes (receptor- $\underline{\text { mediated }}$ endocytosis defective) required for various steps in endocytic transport (Figure 3; Grant and Hirsh, 1999). In contrast to secretory defects, defects in endocytosis per se do not severely affect the organization or morphology of 
the germ line or oocytes as judged by Nomarski optics. The best studied of the general endocytosis regulators identified in this screen are RME-1 and RME-8 (Grant and Hirsh, 1999; Zhang et al., 2001). Both of these proteins are found in the cytoplasm of most $C$. elegans cells and are associated with the limiting membrane of endosomes. RME-1 is an EH domain protein associated with recycling endosomes and is thought to contribute to the formation of membrane tubules that recycle receptors from endosomes to the cell surface (Grant et al., 2001). There are several mammalian homologues of RME-1, at least one of which functions in recycling endosomes (Lin et al., 2001). In the absence of RME-1, yolk receptors become trapped in endosomes of the oocyte and cannot efficiently recycle through multiple rounds of endocytosis. rme-1 mutants are also defective in endocytosis by coelomocytes and in basolateral endocytic traffic in the intestine (Grant et al., 2001).

RME-8 is a large DNA-J domain protein (Zhang et al., 2001). The precise step in trafficking regulated by RME-8 remains unclear, since the protein appears to localize to early and/or late endosomes, but rme- 8 mutants appear defective in the earliest detectable internalization step (Zhang et al., 2001). Recent studies in Drosophila indicate similar localization and transport defects in Dm-rme-8 mutants (Chang et al., 2004). These studies also indicated that RME-8 may interact with Hsc70 and clathrin. J-domains are known to mediate interactions with heat shock protein 70 family members, and clathrin-coated vesicles are uncoated by the action of cytoplasmic Hsc 70 (Brodsky et al., 2001). Previous work in C. elegans demonstrated that yolk endocytosis depends upon the $C$. elegans auxillin homologue $d n j-25$, an other J-domain protein. RNAi of Ce-auxillin results in abnormal accumulation of clathrin-coated structures and interferes with clathrin dynamics in vivo (Greener et al., 2001).

\subsection{Endocytosis in coelomocytes}

The other major model developed for mechanistic studies of endocytosis in C. elegans focuses on coelomocytes, 6 macrophage-like scavenger cells in the body cavity (pseudocoelom) that are highly active in endocytosis of fluid-phase molecules (Fares and Greenwald, 2001). While the natural molecules that are removed from the body cavity by coelomocytes are unknown, coelomocytes are capable of removing many foreign compounds from the body cavity after they are introduced by micro-injection (Fares and Greenwald, 2001). The most common of these endocytosis tracers are fluorescently labeled BSA or dextrans. These observations allowed the development of a pulse-chase type assay of coelomocyte endocytosis (Zhang et al., 2001). Fluorescently labeled BSA or dextran trafficking through the endosomes of coelomocytes is monitored as a function of time after introduction into the body cavity. Expression of RME-8:: GFP is often used to facilitate visualization of endosomes during fluorescent tracer trafficking from the cell surface to the lysosomes (Zhang et al., 2001).

In wild-type animals Texas Red labeled BSA (TR-BSA) molecules endocytosed by coelomocytes first appear in RME- 8 positive endosomes within 5 to 10 minutes after they are introduced into the pseudocoelom. TR-BSA is rapidly concentrated into subendosomal structures, probably membrane buds. The concentrated TR-BSA then appears in LMP-1 positive lysosomes about 30-60 minutes after uptake (Treusch et al., 2004; Zhang et al., 2001).

A simpler and more generally useful assay for coelomocyte endocytic function was developed by Fares and Greenwald (Figure 4; Fares and Greenwald, 2001a; Fares and Greenwald, 2001b). They created transgenic worms expressing signal sequence-GFP fusion protein in body-wall muscles (pmyo-3::ssGFP). In this strain, GFP is constitutively secreted from body-wall muscle cells into the pseudocoelom and is then efficiently taken up and degraded by coelomocytes. At steady-state this strain shows weak pseudocoelom labeling and stronger labeling of late endosome and lysosome structures within coelomocytes. Using this assay, cup (coelomocyte uptake defective) mutants were identified that show accumulation of GFP in the pseudocoelom, and/or aberrant morphology of endocytic organelles within coelomocytes (Figure 4; Fares and Greenwald, 2001b).

Interestingly, all three cup genes cloned so far encode worm homologues of human disease genes. CUP-5 is a homologue of human mucolipin-1, mutations in which lead to Mucolipidosis type IV (Fares and Greenwald, 2001). This family of proteins is thought to act as $\mathrm{Ca}^{2+}$ channels in endosomal or lysosomal membranes. cup-5 mutants show severe defects in lysosome biogenesis that result in the formation of grossly enlarged vacuoles accumulating late endosome, lysosome, and Golgi markers (Treusch et al., 2004). The most likely explanation for this phenotype is that CUP-5 is required for $\mathrm{Ca}^{2+}$ release during the reformation of lysosomes from late endosome-lysosome hybrid organelles. 


\section{ss::GFP endocytosis by coelomocytes}
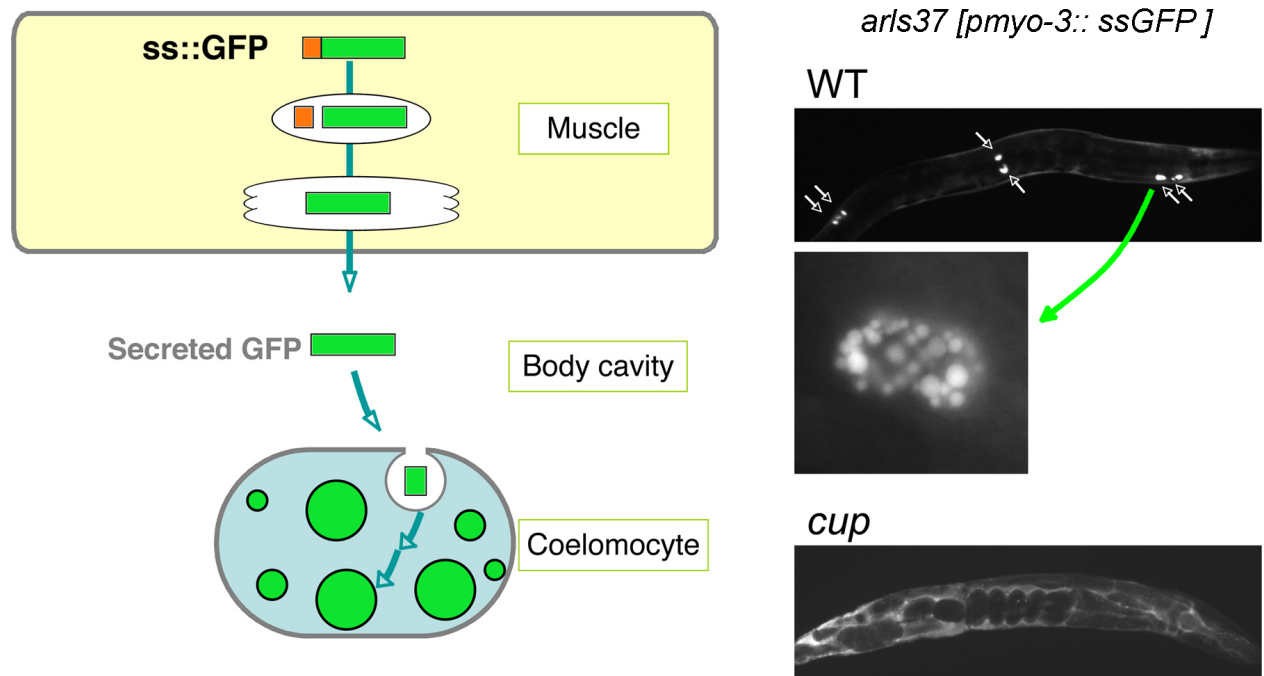

Figure 4. GFP endocytosis by coelomocytes. Signal sequence-tagged GFP is synthesized in muscle and secreted into the body cavity. Coelomocytes efficiently take up GFP from the body cavity and accumulate GFP in large vesicles. Fluorescent micrograph of wild-type and typical cup mutant worms expressing GFP are shown. High magnification image of a wild-type coelomocyte is also shown.

CUP-6/MTM-6 and CUP-10/MTM-9 belong to a family of lipid phosphatases called myotubularins (Dang et al., 2004). Members of this protein family are associated with the human diseases X-linked myotubular myopathy and Charcot-Marie-Tooth type 4B1 and 4B2. MTM-1 and MTM-3, other worm myotubularin homologues, are also required for coelomocyte endocytosis (Xue et al., 2003). Several myotubularins have been demonstrated to specifically dephosphorylate phosphoinositide 3-phosphate [PI(3)P], a key lipid regulator of the endocytic pathway that is normally enriched in early endosomal membranes (Stenmark and Gillooly, 2001). RNAi of C. elegans VPS-34, a homologue of the primary PI-3 kinase, also causes a Cup phenotype, suggesting that the balance of $\mathrm{PI}(3) \mathrm{P}$ production and degradation is important for regulating coelomocyte endocytosis (Fares and Greenwald, 2001b). MTM-6 and MTM-9 form a complex, and both $m t m-6$ and $m t m-9$ mutants show defects in a very early step of endocytosis. Although both MTM-6 and MTM-9 possess the lipid phosphatase domain, MTM-9 lacks key residues thought to be essential for PI-3 phosphatase activity. MTM-9 may play a regulatory role in controlling the phosphatase activity of MTM-6 (Treusch et al., 2004).

It is still unclear to what extent clathrin-dependent endocytosis and clathrin-independent pinocytosis mechanisms contribute to uptake of plasma membrane components and extracellular fluid in coelomocytes. However, dyn-1 and a set of rme mutants (rme-1, -4, -6 and -8) show clear defects in coelomocyte endocytosis in addition to defects in yolk uptake by oocytes (Fares and Greenwald, 2001; Grant et al., 2001; Zhang et al., 2001). These results indicate that the oocyte and coelomocyte pathways share some components. However, oocyte-specific rme mutants and coelomocyte specific cup mutants have been isolated, indicating that not all components are shared between these cells/pathways (Fares and Greenwald, 2001b; our unpublished observation).

\subsection{Signaling and endocytosis}

Signaling receptors are thought to be regulated in part through regulated endocytosis. In some cases endocytosis is thought to down-regulate signaling, while in others endocytosis may be required for signaling receptors to contact their downstream effectors located on endosomal membranes. The LIN-12 receptor is primarily associated with the apical membranes of the VPC cells where it specifies the secondary cell fate (see RTKRas/MAP kinase signaling). The signaling activity of LIN-12 has been shown to be developmentally down-regulated in P6.p by endocytosis (Shaye and Greenwald, 2002). LIN-12 endocytosis is triggered in P6.p by a signal (LIN-3) from the anchor cell received by LET-23 and transduced by Ras (see RTKRas/MAP kinase signaling). Furthermore signaling by the LET-23 EGF receptor is downregulated in P5.p and P7.p in response to the LIN-12 mediated lateral signal, probably in part by increased expression of LET-23 associated endocytosis factors (Yoo et al., 2004). 


\section{The secretory pathway}

Most work on the mechanisms of secretion in $C$. elegans has focused on neuronal cells, and more specifically on the mechanism of synaptic vesicle exocytosis, and to a lesser extent synaptic vesicle biogenesis and axonal transport (see Synaptic function). Much less is known about general or regulated secretion in other $C$. elegans cell types (Figure 5).

\section{Membrane trafficking pathways}

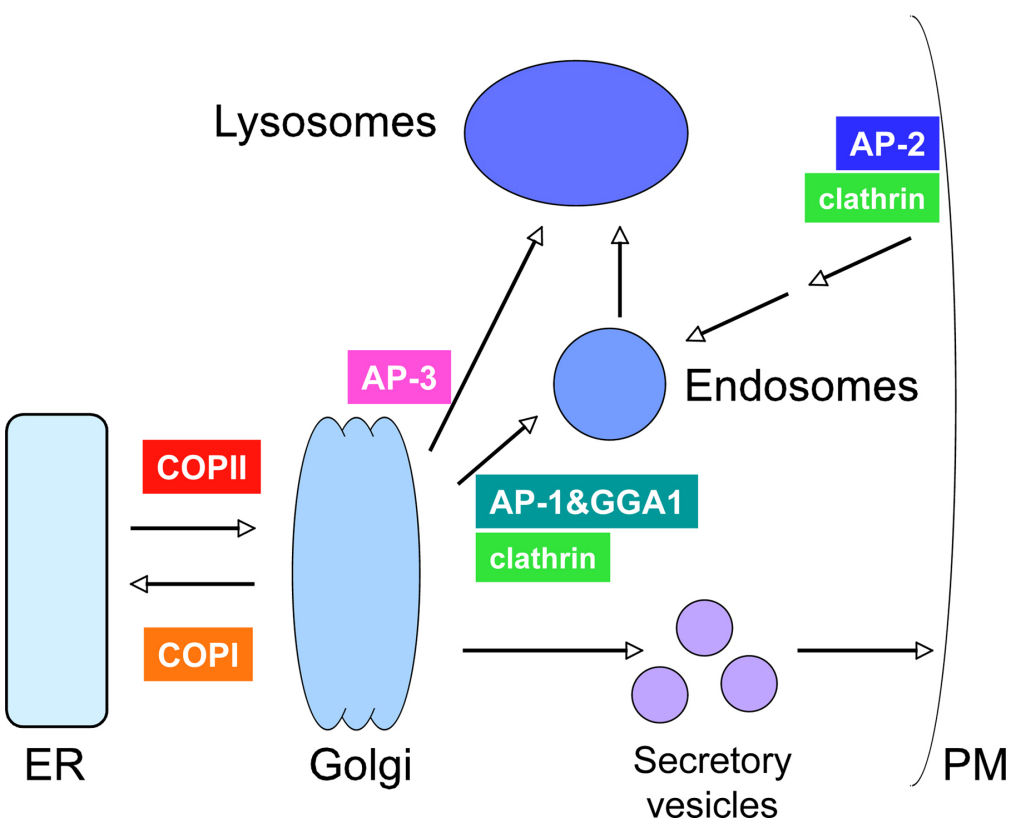

Figure 5. General model of vesicular transport pathways. Transport between organelles is mediated by vesicles. Vesicle formation at various steps is driven by specific coat protein complexes. The $C$. elegans genome contains genes encoding all of these coat proteins, although some of them have not been studied functionally.

As in other eukaryotes $C$. elegans cells possess an endoplasmic reticulum into which most membrane or secretory proteins are inserted co-translationally. Some lumenal ER-resident proteins are retained in the ER by KDEL or HDEL signals in their extreme C-termini. C. elegans cells also possess Golgi stacks, the next organelle through which secretory proteins pass. Unlike mammalian cells, invertebrates such as C. elegans have many small "mini stacks" throughout the cytoplasm of most cells rather than one large stack positioned near the nucleus. From the Golgi, secretory proteins and some membrane proteins proceed to either the plasma membrane or to the endosomal system, depending upon various signals embedded in the primary sequence of the proteins. $C$. elegans does not possess any obvious homologs of the mannose-6-phosphate receptors, and so may not use the mannose-6-phosphate system for tagging and sorting newly synthesized lysosomal hydrolases. Worms may instead use amino acid based signals and a vps10/sortillin receptor type system for such sorting, as is known to be the case in yeast.

\section{General secretion}

Genome-wide RNAi experiments indicate that loss of general secretory factors often results in embryonic lethality and/or larval lethality associated with developmental arrest during molting (Grant and Hirsh, 1999). Analysis in the germline indicates that general secretory defects also result in abnormal oogenesis and often result in multinucleate oocytes, presumably reflecting a defect in cytokinesis (Grant and Hirsh, 1999; Roberts et al., 2003). In addition oocytes lacking normal secretory function can produce embryos lacking a strong eggshell or lacking an eggshell altogether (Grant and Hirsh, 1999). This was not unexpected as the eggshell is thought to form by secretion of eggshell components by the embryo itself shortly after fertilization. 
Several other proteins associated with cytokinesis are known or assumed to function in membrane traffic. These include the endocytic protein dynamin and the syntaxin (t-SNARE) homolog SYN-4 (Jantsch-Plunger and Glotzer, 1999; Thompson et al., 2002). Both of these proteins are associated with cleavage furrows in dividing $C$. elegans germ cells and embryonic cells and are required for completion of cytokinesis (see Cell division). Many proteins associated with the midbody during cytokinesis are thought to have functions in membrane trafficking (Skop et al., 2004).

One component of the general secretory pathway that has been studied in significant detail in C. elegans is SEC-23, a part of the endoplasmic reticulum vesicle coat complex known as COPII (Figure 5). COPII is known to be the primary vesicle coat complex used in yeast and mammalian cell transport from the ER to the Golgi. Roberts et al. identified a single mutant allele of sec-23 in a screen for embryonic lethals defective in cuticle synthesis as assayed by a cuticle collagen reporter, col-12::GFP (Roberts et al., 2003). These authors went on to show severe defects in cuticle synthesis in sec-23 mutants resulting in accumulation of DPY-7 collagen intracellularly, presumably in the ER. Zygotic embryonic lethality during elongation was found in sec-23 homozygous mutants derived from heterozygous mothers. Normal progression through early development presumably relies upon maternally derived SEC-23. RNAi experiments revealed a requirement for sec-23 during larval development, particularly during molting. Adults depleted of SEC-23 by RNAi also showed severe germline defects including binucleate oocytes, lack of yolk uptake by oocytes resulting from a failure of yolk receptors (RME-2) to reach the cell surface, and premature maturation/partitioning of individual germ cells, possibly resulting from loss of cell surface GLP-1 receptors in the distal gonad. A partially functional SEC-23::GFP reporter gene indicated that SEC-23 is broadly expressed at all life stages, and that in hypodermal cells the protein is concentrated in distinct foci. In the embryonic hypodermis these foci were enriched apically at the periphery of the endoplasmic reticulum. These SEC-23 positive foci likely represent ER exit sites where newly synthesized cargo molecules concentrate and are packaged into COPII coated vesicles for delivery to the Golgi.

Another class of C. elegans proteins thought to function in secretion are those related to the putative hedgehog receptor Patched. Kuwabara and colleagues have identified 29 patched (PTC) or patched related (PTR) genes in the C. elegans genome, each of which encodes a predicted multipass transmembrane protein with a predicted sterol-sensing domain (Kuwabara et al., 2000). The phenotypes of ptc-l mutants indicate a likely defect in the secretory pathway and in germline cytokinesis in particular. The presence of such a large and diverse family of these proteins in C. elegans could indicate diverse functions in membrane trafficking processes (Kuwabara et al., 2000).

\section{Polarized secretion}

Polarized cells such as epithelia maintain distinct apical and basolateral plasma membrane domains with distinct protein and lipid compositions. These specializations of the plasma membrane require complex membrane trafficking pathways thought to include unique basolateral versus apical sorting mechanisms in the secretory pathway to direct newly synthesized proteins to the correct membrane domain. Additional regulation of the endocytosis pathway maintains polarity of lipid and protein components after internalization and recycling.

Ectodermal cells with epithelial characteristics are an important class of polarized cells that have come under intense scrutiny in C. elegans in recent years. These include epidermal cells (hypodermal cells), vulval and rectal epithelia, glia-like sheath and socket cells that support sensory neurons of the amphid and phasmid sensory organs, and the excretory cell, a single cell that forms a renal-type organ, extending a long cytoplasmic process called the excretory canal on each side of the animal.

\subsection{Apical secretion}

A large number of mutants (exc genes) that display defects in apical membrane formation in excretory cells have been identified (Buechner et al., 1999). Some of these are likely to function directly in membrane transport to form or maintain apical membranes. A likely candidate for mediating vesicular transport is EXC-4, a member of the CLIC chloride channel family (Berry et al., 2003). EXC-4 localizes to vesicles that fuse together to form the apical membrane of the lumen in the excretory canals. Furthermore exc-4 is continuously required for tube formation and maintenance. Although the precise mechanisms remain unclear, EXC-4 may function in apical secretory vesicle fusion to promote tube formation.

All of the ectodermal epithelial-like cells are known to require the lin-26 transcription factor to aquire their final differentiated forms (Labouesse et al., 1994). In particular it is the apical membrane domain that appear most 
severely affected in lin-26 mutant ectodermal epithelia. LIN-26 has therefore been proposed to regulate the expression of gene products that form and maintain apical epithelial character. Screens for mutants with Lin-26-like defects could therefore identify apical-specific trafficking factors. Michaux et al (Michaux et al., 2000) took just this approach and identified CHE-14, a predicted 12-pass transmembrane protein required for the function of these cell-types. che-14 mutants displayed defects in the hypodermis, excretory canal, vulva, rectum and amphids and phasmids. In che-14 mutants vesicles and amorphous material accumulate near the apical surface of the affected cells suggesting that secretion is defective. CHE-14 contains a sterol-sensing domain, a type of domain found in proteins such as Dispatched, Patched and PC1 that are involved in cholesterol associated trafficking processes. A rescuing CHE-14::GFP reporter is localized to the apical surface of epithelial cells that require che-14 function, consistent with such a proposed function (Michaux et al., 2000).

\subsection{Basolateral secretion}

Basolateral-specific transport pathways are also thought to play key roles in polarized cells. The best studied of these pathways in C. elegans is in the transport of the LET-23 EGF-receptor in the vulval precursor cells (VPCs; see RTKRas/MAP kinase signaling). LET-23 is normally restricted to the basolateral membrane of the VPCs, a process that requires the PDZ-domain proteins LIN-2, LIN-7, and LIN-10 (Kaech et al., 1998). The LIN-2/7/10 complex binds directly to the LET-23 intracellular domain, and is therefore likely to function as a sorting factor, directing LET-23 to the basolateral membrane during secretion and/or after endocytosis and recycling (Kaech et al., 1998). The LIN-10 protein has been shown to associate with an intracellular compartment on or near the Golgi consistent with a role in protein sorting during secretion (Whitfield et al., 1999).

\section{References}

Berry, K.L., Bulow, H.E., Hall, D.H., and Hobert, O. (2003). A C. elegans CLIC-like protein required for intracellular tube formation and maintenance. Science 302, 2134-2137. Abstract Article

Brodsky, F.M., Chen, C.Y., Knuehl, C., Towler, M.C., and Wakeham, D.E. (2001). Biological basket weaving: formation and function of clathrin-coated vesicles. Annu. Rev. Cell Dev. Biol. 17, 517-568. Abstract Article

Buechner, M., Hall, D.H., Bhatt, H., and Hedgecock, E.M. (1999). Cystic canal mutants in Caenorhabditis elegans are defective in the apical membrane domain of the renal (excretory) cell. Dev. Biol. 214, 227-241. Abstract Article

Chang, H.C., Hull, M., and Mellman, I. (2004). The J-domain protein Rme-8 interacts with Hsc70 to control clathrin-dependent endocytosis in Drosophila. J. Cell Biol. 164, 1055-1064. Abstract Article

Dang, H., Li, Z., Skolnik, E.Y., and Fares, H. (2004). Disease-related myotubularins function in endocytic traffic in Caenorhabditis elegans. Mol. Biol. Cell 15, 189-196. Abstract Article

Fares, H., and Grant, B. (2002). Deciphering endocytosis in Caenorhabditis elegans. Traffic 3, 11-19. Abstract Article

Fares, H., and Greenwald, I. (2001a). Genetic analysis of endocytosis in Caenorhabditis elegans: coelomocyte uptake defective mutants. Genetics 159, 133-145. Abstract Article

Fares, H., and Greenwald, I. (2001b). Regulation of endocytosis by CUP-5, the Caenorhabditis elegans mucolipin-1 homolog. Nat. Genet. 28, 64-68. Abstract Article

Grant, B., and Hirsh, D. (1999). Receptor-mediated endocytosis in the Caenorhabditis elegans oocyte. Mol. Biol. Cell 10,4311-4326. Abstract

Grant, B., Zhang, Y., Paupard, M.C., Lin, S.X., Hall, D.H., and Hirsh, D. (2001). Evidence that RME-1, a conserved C. elegans EH-domain protein, functions in endocytic recycling. Nat. Cell Biol. 3, 573-579. Abstract Article

Greener, T., Grant, B., Zhang, Y., Wu, X., Greene, L.E., Hirsh, D., and Eisenberg, E. (2001). Caenorhabditis elegans auxilin: a J-domain protein essential for clathrin-mediated endocytosis in vivo. Nat. Cell Biol. 3, $215-219$. Abstract Article 
Jantsch-Plunger, V., and Glotzer, M. (1999). Depletion of syntaxins in the early Caenorhabditis elegans embryo reveals a role for membrane fusion events in cytokinesis. Curr. Biol. 9, 738-745. Abstract

Kaech, S.M., Whitfield, C.W., and Kim, S.K. (1998). The LIN-2/LIN-7/LIN-10 complex mediates basolateral membrane localization of the $C$. elegans EGF receptor LET-23 in vulval epithelial cells. Cell 94, 761-771. Abstract Article

Kuwabara, P.E., Lee, M.H., Schedl, T., and Jefferis, G.S. (2000). A C. elegans patched gene, ptc-1, functions in germ-line cytokinesis. Genes Dev. 14, 1933-1944. Abstract

Labouesse, M., Sookhareea, S., and Horvitz, H.R. (1994). The Caenorhabditis elegans gene lin-26 is required to specify the fates of hypodermal cells and encodes a presumptive zinc-finger transcription factor. Development 120 , 2359-2368. Abstract

Lin, S.X., Grant, B., Hirsh, D., and Maxfield, F.R. (2001). RME-1 regulates the distribution and function of the endocytic recycling compartment in mammalian cells. Nat. Cell. Biol. 3, 567-572. Abstract Article

Michaux, G., Gansmuller, A., Hindelang, C., and Labouesse, M. (2000). CHE-14, a protein with a sterol-sensing domain, is required for apical sorting in C. elegans ectodermal epithelial cells. Curr. Biol. 10, 1098-1107. Abstract

Roberts, B., Clucas, C., and Johnstone, I.L. (2003). Loss of SEC-23 in Caenorhabditis elegans causes defects in oogenesis, morphogenesis, and extracellular matrix secretion. Mol. Biol. Cell 14, 4414-4426. Abstract Article

Shaye, D.D., and Greenwald, I. (2002). Endocytosis-mediated downregulation of LIN-12/Notch upon Ras activation in Caenorhabditis elegans. Nature 420, 686-690. Abstract Article

Skop, A.R., Liu, H., Yates, J., III, Meyer, B.J., Heald, R. (2004). Dissection of the mammalian midbody proteome reveals conserved cytokinesis mechanisms. Science 305, 61-66. Abstract Article

Stenmark, H., and Gillooly, D.J. (2001). Intracellular trafficking and turnover of phosphatidylinositol 3-phosphate. Semin. Cell Dev. Biol. 12, 193-199. Abstract Article

Thompson, H.M., Skop, A.R., Euteneuer, U., Meyer, B.J., and McNiven, M.A. (2002). The large GTPase dynamin associates with the spindle midzone and is required for cytokinesis. Curr. Biol. 12, 2111-2117. Abstract Article

Treusch, S., Knuth, S., Slaugenhaupt, S.A., Goldin, E., Grant, B.D., and Fares, H. (2004). Caenorhabditis elegans functional orthologue of human protein h-mucolipin-1 is required for lysosome biogenesis. Proc. Natl. Acad. Sci. USA 101, 4483-4488. Abstract Article

Whitfield, C.W., Benard, C., Barnes, T., Hekimi, S., and Kim, S.K. (1999). Basolateral localization of the Caenorhabditis elegans epidermal growth factor receptor in epithelial cells by the PDZ protein LIN-10. Mol. Biol. Cell 10, 2087-2100. Abstract

Xue, Y., Fares, H., Grant, B., Li, Z., Rose, A.M., Clark, S.G., and Skolnik, E.Y. (2003). Genetic analysis of the myotubularin family of phosphatases in Caenorhabditis elegans. J. Biol. Chem. 278, 34380-34386. Abstract Article

Yoo, A.S., Bais, C., Greenwald, I. (2004). Crosstalk between the EGFR and LIN-12/Notch pathways in C. elegans vulval development. Science 303, 663-666. Abstract Article

Zhang, Y., Grant, B., and Hirsh, D. (2001). RME-8, a conserved J-domain protein, is required for endocytosis in Caenorhabditis elegans. Mol. Biol. Cell 12, 2011-2021. Abstract

All WormBook content, except where otherwise noted, is licensed under a Creative Commons Attribution License 